\title{
Adaptation to Vocal Expressions Reveals Multistep Perception of Auditory Emotion
}

\author{
Patricia E. G. Bestelmeyer, ${ }^{1}$ Pierre Maurage, ${ }^{2}$ Julien Rouger, ${ }^{3}$ Marianne Latinus, ${ }^{4}$ and Pascal Belin ${ }^{4,5,6}$ \\ ${ }^{1}$ School of Psychology, Bangor University, Bangor, Gwynedd LL57 2AS, United Kingdom, ${ }^{2}$ Cognitive Neurosciences and Clinical Psychology Research Units, \\ Department of Psychology, Catholic University of Louvain, 1348 Louvain-la-Neuve, Belgium, ${ }^{3}$ Department of Cognitive Neuroscience, University of \\ Maastricht, 6200 MD, Maastricht, The Netherlands, ${ }^{4}$ Institut de Neurosciences de La Timone, Unité Mixte de Recherche 7289, Aix-Marseille Université, \\ Centre National de la Recherche Scientifique, 13385 Marseille, France, ${ }^{5}$ Institute of Neuroscience and Psychology, University of Glasgow, Glasgow G12 8QB, United \\ Kingdom, and ' International Laboratory for Brain, Music and Sound Research, University of Montréal/McGill University, Montréal H3C 3J7, Canada
}

The human voice carries speech as well as important nonlinguistic signals that influence our social interactions. Among these cues that impact our behavior and communication with other people is the perceived emotional state of the speaker. A theoretical framework for the neural processing stages of emotional prosody has suggested that auditory emotion is perceived in multiple steps (Schirmer and Kotz, 2006) involving low-level auditory analysis and integration of the acoustic information followed by higher-level cognition. Empirical evidence for this multistep processing chain, however, is still sparse. We examined this question using functional magnetic resonance imaging and a continuous carry-over design (Aguirre, 2007) to measure brain activity while volunteers listened to non-speech-affective vocalizations morphed on a continuum between anger and fear. Analyses dissociated neuronal adaptation effects induced by similarity in perceived emotional content between consecutive stimuli from those induced by their acoustic similarity. We found that bilateral voice-sensitive auditory regions as well as right amygdala coded the physical difference between consecutive stimuli. In contrast, activity in bilateral anterior insulae, medial superior frontal cortex, precuneus, and subcortical regions such as bilateral hippocampi depended predominantly on the perceptual difference between morphs. Our results suggest that the processing of vocal affect recognition is a multistep process involving largely distinct neural networks. Amygdala and auditory areas predominantly code emotion-related acoustic information while more anterior insular and prefrontal regions respond to the abstract, cognitive representation of vocal affect.

Key words: fMRI; vocal emotion; voice perception

\section{Introduction}

Voices convey an array of social information such as the gender, identity, and emotional state of the speaker. Amplitude, fundamental frequency, and voice smoothness are only some of the variables that convey emotion by physiological changes in the vocal production system (Patel et al., 2011). Perceiving these acoustic cues as a complex message that communicates emotions or intentions even in the absence of speech is crucial for everyday social interactions (Wallbott and Scherer, 1986). Research on auditory affect perception has concentrated on the perception of speech prosody with a neuroscientific model pro-

Received Nov. 16, 2013; revised April 15, 2014; accepted April 23, 2014.

Author contributions: P.E.G.B. and P.B. designed research; P.E.G.B. and P.M. performed research; J.R., M.L., and P.B. contributed unpublished reagents/analytic tools; P.E.G.B. analyzed data; P.E.G.B. wrote the paper.

This research was supported by Economic and Social Research Council/Medical Research Council Grant RES-06025-0010.

The authors declare no competing financial interests.

This article is freely available online through the J Neurosci Author Open Choice option.

Correspondence should be addressed to Dr. Patricia E.G. Bestelmeyer, School of Psychology, Bangor University, Gwynedd LL57 2AS, UK. E-mail: p.bestelmeyer@bangor.ac.uk.

DOI:10.1523/JNEUROSCI.4820-13.2014

Copyright (c) 2014 Bestelmeyer et al.

This is an Open Access article distributed under the terms of the Creative Commons Attribution License (http://creativecommons.org/licenses/by/3.0), which permits unrestricted use, distribution and reproduction in any medium provided that the original work is properly attributed. posing the anatomical underpinnings that are involved (Schirmer and Kotz, 2006). The first stage of this model consists of low-level acoustic analyses in bilateral auditory cortices. These areas then project to superior temporal sulci (STSs) and superior temporal gyri (STGs) for more complex processing in which emotionally salient information is synthesized into an emotional "Gestalt" or acoustic object. The STS and STG then feed into frontal areas for higher-order cognition (e.g., evaluative judgments of prosody). This model provides an analytical first step toward understanding the network involved, but more research is necessary to test its predictions and expand it to the perception of other emotional auditory objects.

We were interested in examining the neural processing stages of vocal affect perception using a specific type of adaptation paradigm. Behaviorally, adaptation refers to a process during which continued stimulation results in biased perception toward opposite features of the adaptor (Grill-Spector et al., 1999). These aftereffects have been demonstrated behaviorally for vocal affect perception (Bestelmeyer et al., 2010; Skuk and Schweinberger, 2013). Neurally, fMRI adaptation to a specific stimulus feature is typically accompanied by a decrease in the hemodynamic response (also referred to as repetition suppression). Adaptation paradigms are often used to reveal neural populations tuned to the processing of a specific stimulus attribute (i.e., to reveal func- 
tional specificity of neural populations). Continuous carry-over designs present an unbroken stream of serially balanced stimuli, thereby allowing the evaluation of adaptation or "carry-over" effects between subsequent stimuli (Aguirre, 2007).

We created morph continua between angry and fearful vocalizations (Bestelmeyer et al., 2010). Carry-over effects between consecutive stimuli were assessed by calculating physical and perceptual differences between morphs in the stimulus sequence and entering these values as regressors in a general linear model. The "physical difference" regressor consisted of the absolute difference in morph step between a given stimulus and the preceding one, directly proportional to the acoustic difference. The "perceptual difference" regressor consisted of the absolute difference in proportion of fear responses between stimuli and was orthogonalized with respect to the "physical regressor" in the design matrix. This design allowed us to examine adaptation effects induced by perceived emotion after variance accounted for by acoustic differences had been explained. We hypothesized that carry-over effects to the physical properties would be evident in voice-sensitive areas with a lateralization toward the right (Belin et al., 2000; Lewis et al., 2009; Bestelmeyer et al., 2011). In contrast, we expected the cognitive representations of emotion to engage separate, higher-level areas involving frontal cortices.

\section{Materials and Methods}

Participants. Nineteen healthy volunteers from the under- and postgraduate community of the University of Glasgow took part (13 females, mean age $=22.63$, range $=18-32$ years, $S D=3.96$ ). All participants were of normal hearing (according to a brief audiometric test) and were reimbursed $£ 12$ for their time. Informed consent was obtained from all individuals and the study protocol was approved by the local ethics committee.

Experimental stimuli and $f M R I$ paradigm. Recordings were taken from the Montreal Affective Voices (Belin et al., 2008) in which actors were instructed to produce emotional interjections using the vowel /a/. The voices from six identities (three female) expressed anger and fear. Angry to fearful continua were created separately for each identity in seven steps that corresponded to (fear/anger) 5/95\%, 20/80\%, 35/65\%, 50/50\%, 65/ $35 \%, 80 / 20 \%$ and $95 / 5 \%$. The duration of the vocalizations within each continuum was kept constant and ranged between 0.6 and $0.9 \mathrm{~s}$ across continua. We used STRAIGHT for stimulus generation (Kawahara and Matsui, 2003; for more information, see also http://www.wakayama-u. ac.jp/ kawahara/STRAIGHTadv/index_e.html). STRAIGHT performs an instantaneous pitch-adaptive spectral smoothing of each stimulus for separation of contributions to the voice signal arising from the glottal source [including the perceived pitch of the voice (fo)] versus supralaryngeal filtering [distribution of spectral peaks, including the first formant frequency (F1)]. Voice stimuli were decomposed by STRAIGHT into the following five parameters: f0, frequency, duration, spectrotemporal density, and aperiodicity. Each parameter can be manipulated independently. For each voice, we manually identified one time landmark with three frequency landmarks (corresponding to the first three formants) at the onset of phonation and the same number of landmarks at the offset of phonation. Morphed stimuli were then generated by resynthesis based on the interpolation (linear for time; logarithmic for F0, frequency, and amplitude) of these time-frequency landmark templates (for a recent discussion of the voice morphing technique, see also Schweinberger et al., 2014). Stimuli were normalized in energy (root mean square) before and after morphing. Acoustic analyses to illustrate the validity of the morphing technique with affective bursts have been published previously (Bestelmeyer et al., 2010). We used Psychtoolbox-3 (Brainard, 1997; Pelli, 1997) for stimulus presentation and response recording. Both programs were run in MatlabR2007b (MathWorks).

We used a continuous carry-over design (Aguirre, 2007) to measure the effects of one stimulus upon the next using a first-order serially balanced sequence of stimuli known as type-1-index-1 (Nonyane and
Theobald, 2007). Our carry-over sequence was defined by eight items: the seven morph steps of a continuum (always within identity) plus one silent null event. One complete carry-over sequence with eight items comprises 65 stimuli. In this balanced sequence, each stimulus is preceded and followed by every other stimulus an equal number of times. Thus, every item is repeated eight times per sequence (except for the first item, which is repeated nine times; for a sample sequence of eight items, see http://www.bioss.ac.uk/people/cmt/seqlist.html\#08). The end of each carry-over sequence was followed by nine TRs of silences (18 s) before the start of the next carry-over sequence. Each of our six identities was presented once per run, leading to six different carry-over sequences and totaling 390 stimuli per run. Gender was blocked so that an experimental run started with three male carry-over sequences followed by three female carry-over sequences or vice versa. We acquired three experimental fMRI runs.

Sounds were presented binaurally using the electrostatic NNL headphone system (NordicNeuroLab) at an intensity of $80 \mathrm{~dB}$ SPL(C). Participants were asked to perform a two-alternative forced choice task in which each affective voice had to be categorized as either angry or fearful by means of two buttons mounted on an MRI-compatible response box (fORP, Current Designs). Participants were asked to react as fast as possible and to keep their eyes closed for the duration of all scans. For each participant, the behavioral responses were used to compute the perceptual difference regressor, which was then fed into the parametric modulation analyses. Following this procedure, we administered a short "voice localizer" scan (for details, see Belin et al., 2000) to localize areas that are particularly sensitive to voices compared with other, environmental sounds.

Image acquisition and analysis. All scans were acquired in a 3.0 tesla Siemens Tim Trio scanner using a 12-channel head coil. Whole-brain T1-weighted anatomical scans were performed using fast gradient echo known as T1 magnetization-prepared rapid acquisition gradient echo, consisting of 192 axial slices of $1 \mathrm{~mm}$ thickness with an in-plane resolution of $1 \times 1 \times 1$ [field of view $(\mathrm{FOV})=256^{\circ}$ ] and a matrix of $256 \times 256$ performed at the end of the experimental session. $\mathrm{T} 2^{*}$-weighted functional scans were acquired using an interleaved ascending sequence consisting of 32 slices of $3 \mathrm{~mm}$ thickness $(0.3 \mathrm{~mm}$ gap) with an in-plane resolution of $3 \times 3 \times 3\left(\mathrm{FOV}=1260^{\circ}\right)$, an acquisition matrix of $70 \times 70$, and a flip angle of $77^{\circ}$. The three runs of the experimental scan (TR $=2 \mathrm{~s}$, $\mathrm{TE}=30 \mathrm{~ms}$ ) consisted of 446 volumes each, while the voice localizer $(\mathrm{TR}=2 \mathrm{~s} ; \mathrm{TE}=30 \mathrm{~ms})$ scan consisted of one run of 310 volumes and allows reliable identification of the temporal voice areas by contrasting vocal with nonvocal sounds.

All MRI data were analyzed using SPM8 (Wellcome Department of Cognitive Neurology, University College London, http://www.fil.ion. ucl.ac.uk/spm/). Preprocessing of the data consisted of anterior commissure-posterior commissure alignment of the anatomical images (and application of the orientation change to all functional images acquired in the same session). Functional scans were corrected for head motion (trilinear interpolation) by aligning all scans to the first scan of the last run, and a mean image was created. The anatomical scan was coregistered to the mean image. Functional and anatomical data were transformed to Montreal Neurological Institute space after segmentation of the anatomical scan. Normalized data were spatially smoothed by applying a Gaussian kernel of $8 \mathrm{~mm}$ full-width at half-maximum.

We used parametric modulations to analyze our data (Büchel et al., 1998). We linearly rescaled the parametric variables so that all measurements were on a comparable scale lying between 0 and 1 using the "MinMax" normalization. This transformation preserves the relationships among the original data values and ensures that the regressors have equivalent weights. For each auditory stimulus (except the first sound in each carry-over sequence), we calculated the physical and perceptual differences between it and the preceding stimulus (Fig. 1). The design matrix of first-level analyses contained all voice onset events as the first regressor which was followed by four parametric regressors, as follows: (1) "first voice" of each block (to remove the effect of a stimulus following baseline); (2) "morph step" (i.e., the percentage of fearfulness contained in the voice); (3) physical difference, which was computed as the absolute difference in morph step between two consecutive stimuli; and 


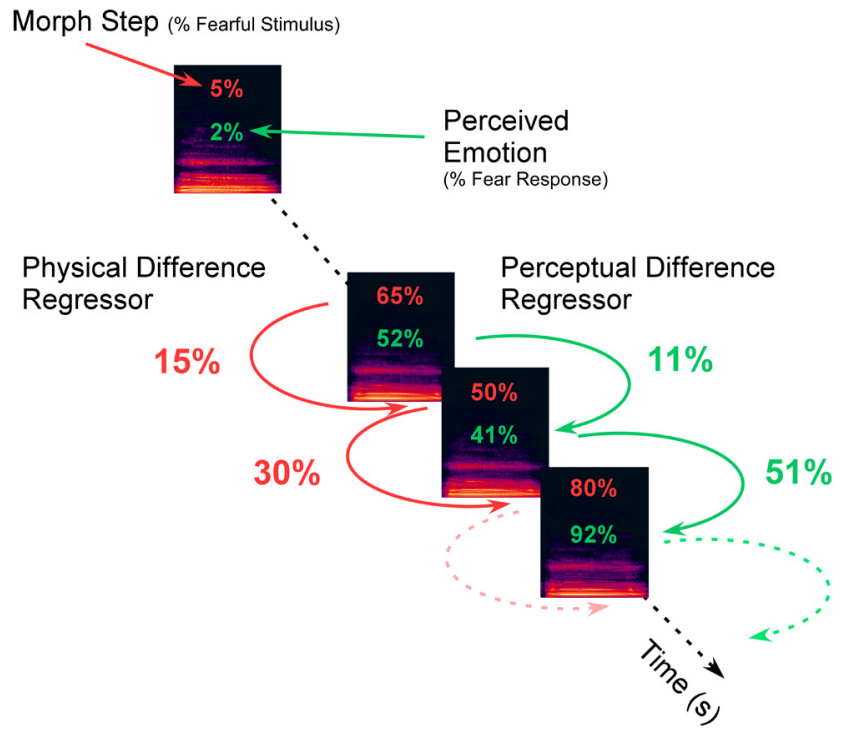

Figure 1. Illustration of the calculation of the physical (red) and perceptual (green) difference regressors. The physical difference regressor is calculated based on the absolute difference in morph step between two consecutively presented stimuli. Similarly, the perceptual difference regressor is calculated based on the absolute difference in the averaged participant's behavioral responses (two alternative forced choice responses) to each morph step between two consecutively presented stimuli.
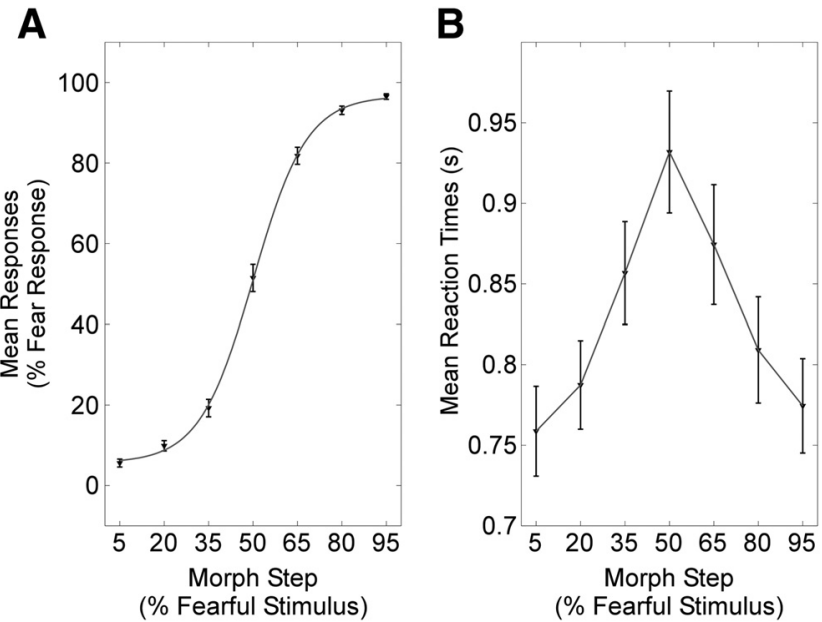

Figure 2. $\quad \boldsymbol{A}$, Results of the emotion categorization task. $\boldsymbol{B}$, Reaction time as a function of morph step. Error bars represent the SEM.

(4) perceptual difference, which was calculated in the same way as regressor 2 but was based on the participant's behavioral responses (Fig. 2; Aguirre, 2007; Charest et al., 2013). Thus, for the perceptual difference regressor of each run, we calculated the mean behavioral response to each morph step and then calculated the absolute difference in mean responses between two consecutive stimuli. The perceptual difference regressor was therefore based on the participant's own subjective judgments of the affective morphs. Figure 1 illustrates how the regressors for the physical difference (red) and perceptual difference (green) were calculated.

Note that each of the four parametric regressors was orthogonalized (i.e., rendering collinear regressors linearly independent) with respect to the previous ones to account only for variance not explained by previous regressors. We also included the silent null events presented within the carry-over sequences and six movement parameters in our model. Second-level analysis of the morph step and physical difference regressors consisted of one-sample $t$ tests for each regressor, allowing for the evaluation of positive and negative correlations. The analysis of the per-
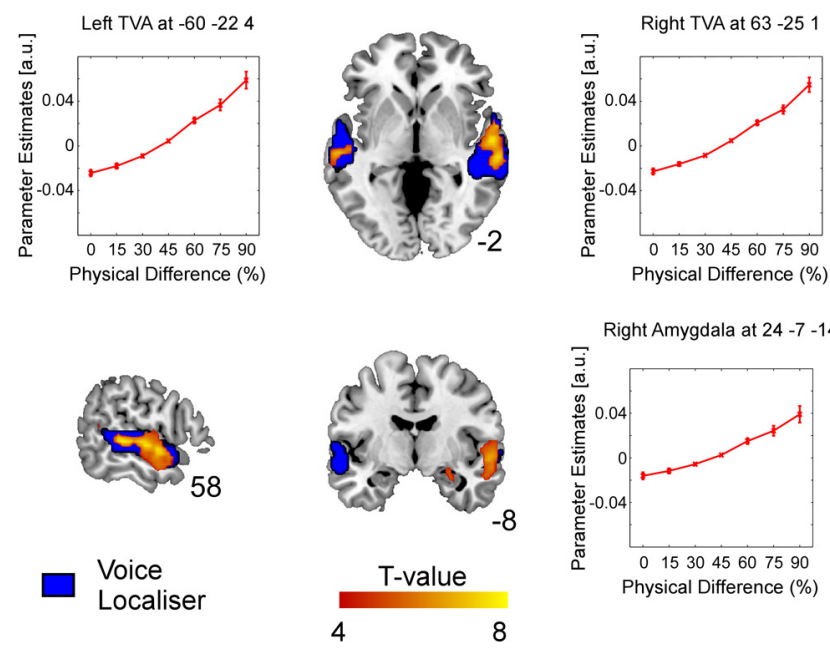

Figure 3. Activation maps of significant correlations between the physical difference between consecutive stimuli and BOLD signal illustrated on a T1-weighted average structural template. Positive correlations (i.e., increased neuronal adaptation for more similar consecutive stimuli) are evident in areas that overlap with bilateral voice-sensitive cortex (shown in blue) and right amygdala (see also associated plots of parameter estimates in the peak voxels of these clusters).

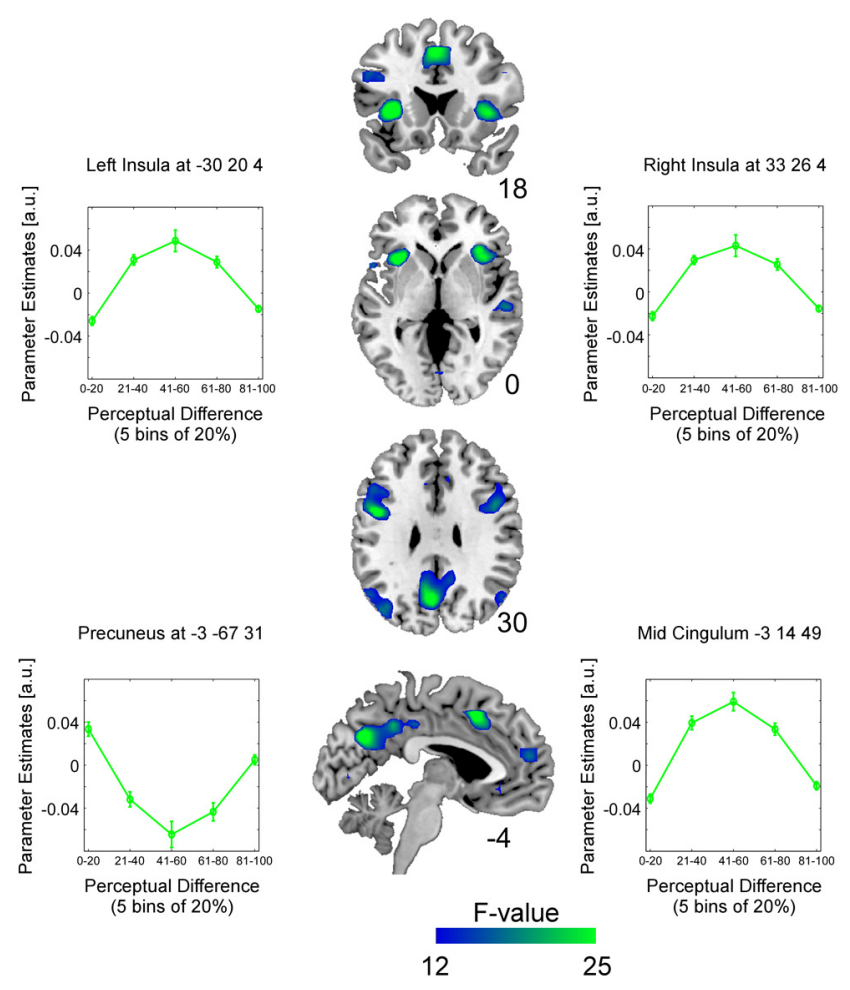

Figure 4. Activation maps of significant correlations between perceptual difference between morph steps and BOLD signal illustrated on a T1-weighted average structural template. The $F$-contrast combines linear and quadratic expansions of the perceptual regressor. Significant negative quadratic relationships are evident in bilateral insulae, mid-cingulum, medial orbitofrontal and superior frontal gyri, as well as bilateral precentral gyri. A positive quadratic relationship was obtained in bilateral precuneus.

ceptual difference regressor consisted of an $F$ test across linear and quadratic contrasts in a full-factorial ANOVA with two levels (level 1: $\mathrm{T}$-contrast images from the linear expansion; level 2: T-contrast images from the quadratic expansion). To illustrate the parametric modulations in Figures 3 and 4, we used the built-in function of SPM8 (spm_graph.m) to extract the $\beta$ estimates at the peak maxima within a sphere of $6 \mathrm{~mm}$. 
Unlike the physical difference, behavioral responses did not average to discrete steps but, rather, resulted in a range of numbers. Therefore, to account for the interindividual variability of behavioral responses and to allow the averaging across participants, we grouped averaged behavioral responses into five bins of $20 \%$. Results are illustrated on an average anatomical scan using MRIcron (Rorden et al., 2007). Anatomical cluster location was assessed with xjview version 8.1 (http://www.alivelearn. net/xjview) and cross-checked with the brain atlas of Duvernoy (1999) to ensure accuracy.

Our two regressors of interest (physical and perceptual difference) are not naturally orthogonal. Given our design, the physical regressor, entered before the perceptual regressor, may therefore contain variance that is shared by both regressors (i.e., variance that is neither purely physical nor purely perceptual). We therefore computed two further "single" models for each participant, as follows: (1) a model with the physical regressor but without the perceptual regressor; and (2) a model with the perceptual regressor but without the physical regressor. These models were otherwise identical to the one reported above.

\section{Results}

\section{Behavioral results}

Results of the behavioral data are summarized in Figure 2. The response data were averaged as a function of the seven morph steps, and a psychophysical curve (based on the hyperbolic tangent function) was fitted to the mean data (Fig. 2A; see also Bestelmeyer et al. (2010) for an identical pattern obtained without scanner noise). Participants more frequently categorized the first three morph steps as anger and the last three morph steps as fear, while the 50\% morph was perceived as the most emotionally ambiguous. Reaction times were lowest for the least ambiguous stimuli and highest for the 50\% morph with $>150 \mathrm{~ms}$ difference between extreme versus central points of the continuum (Fig. 2B).

\section{fMRI results}

We used parametric modulation analyses to investigate areas that respond to a modulation of the morph step, physical difference, and then perceptual difference from a combined general linear model (see $(A)$ and $(B)$, below). All results are illustrated at a threshold of $p<0.0001$ (uncorrrcted) and an extent threshold of 30 voxels. Unless stated otherwise, statistical significance was assessed at the cluster level with a threshold of $p<0.05$ and was FWE corrected for multiple comparisons across the whole brain. No significant modulations with morph step were observed (i.e., no brain area reacted more to anger than to fear and vice versa).

\section{(A) Carry-over effect of the physical difference between} consecutive morphs

Parametric modulation analysis of the physical difference regressors showed significant positive correlations between physical difference and BOLD signal in bilateral superior/midtemporal gyri (right: $t_{(18)}=8.35, k=491$; left: $t_{(18)}=7.52, k=161$ ), right amygdala $\left(t_{(18)}=5.90, k=32\right)$ and a small cluster covering mid-cingulum $\left(t_{(18)}=6.27, k=36\right)$. This neuronal adaptation is illustrated in Figure 3, and significant clusters are summarized in Table 1. In these regions, activity was greater in response to stimuli that were more acoustically different from the preceding stimulus (larger morph step difference).

\section{(B) Carry-over effect of the perceptual difference between consecutive morphs}

The perceptual difference between morphs was based on the absolute difference between averaged proportions of fear responses to each morph (not averaged across the group but specific to each participant) and was included as an orthogonalized regressor, after variance explained by physical differences between stimuli had been accounted for. The adaptation framework predicts a (typically linear) decrease in BOLD signal with stimulus repetition. However, previous research has shown quadratic relationships between perceptual differences and BOLD signal (Charest et al., 2013). We therefore included linear and quadratic expansions of the perceptual difference regressor. To test for a significant effect of modulations in perceptual difference, we calculated an $F$-contrast to combine linear and quadratic expansions of this regressor. This analysis revealed significant negative quadratic relationships in bilateral anterior insulae with inferior frontal gyri (IFGs; right: $F_{(2,36)}=28.78, k=169$; left: $F_{(2,36)}=38.80, k=$ 153 ), bilateral precentral gyri (right: $F_{(2,36)}=25.28, k=240$; left: $F_{(2,36)}=37.75, k=336$ ), bilateral middle temporal gyrus (MTG), superior temporal gyrus (STG; right STG: $F_{(2,36)}=21.52, k=75$; left STG: $F_{(2,36)}=25.34, k=131$; left MTG: $F_{(2,36)}=23.73, k=$ $241)$, supplementary motor area/mid-cingulum $\left(F_{(2,36)}=32.90\right.$, $k=205)$, left superior frontal region $\left(F_{(2,36)}=25.20, k=66\right)$, left medial superior frontal $\left(F_{(2,36)}=19.40 ; k=98\right)$, right lingual $\left(F_{(2,36)}=20.21, k=83\right)$, and a positive quadratic relationship in a cluster covering bilateral precuneus $\left(F_{(2,36)}=32.44, k=516\right)$.

At a more lenient threshold of $\mathrm{p}<0.001$ (uncorrected), using small-volume corrections ( $6 \mathrm{~mm})$, a number of subcortical structures survived FEW correction at the peak level (i.e., clusters covering bilateral hippocampi and amygdalae; right: $F_{(2,36)}=$ 20.51, $k=19$, MNI coordinates $=24-4-23$; left: $F_{(2,36)}=$ $14.09, k=20$, MNI coordinates $=-24-7-20)$ and thalami (right: $F_{(2,36)}=16.38, k=29$, MNI coordinates $=12-194$; left: $F_{(2,36)}=17.02, k=28, \mathrm{MNI}$ coordinates $\left.=-12-164\right)$. Figure 3 illustrates significant clusters and illustrations of the parameter estimates in bilateral anterior insulae, mid-cingulum, and precuneus. Significant clusters are summarized in Table 1.

We then computed parametric modulation analyses of two single general linear models (reported in $(C)$, below) to address whether regions dealing with physical and perceptual differences of vocal expressions involve partially overlapping regions.

\section{(C) Overlap between the carry-over effect for the physical and perceptual difference from the two single models}

Parametric modulation analyses of the two single models (either with the physical but not the perceptual regressor or vice versa) showed very similar patterns of activations to our combined model reported above. The analyses confirm that while the two steps necessary for the acoustic and perceptual processing of vocal emotion are largely distinct, there is some overlap between the two processes in right midtemporal and left superior temporal gyrus and left putamen (Fig. 5). Significant clusters of both single models are summarized in Table 2.

\section{Discussion}

We were interested in disentangling the different regions of the vocal emotion network that process acoustic differences between emotional vocalizations from regions that deal with the cognition and evaluation of vocally expressed emotions by using a new adaptation design (Aguirre, 2007). We morphed nonverbal expressions on a continuum between anger and fear, and presented the stimuli in a continuous and balanced sequence (Nonyane and Theobald, 2007). We found linear neuronal adaptation effects in response to the physical difference between two consecutively presented voice morphs in bilateral voice-sensitive cortices (Belin and Zatorre, 2000; Lewis et al., 2009; Bestelmeyer et al., 2011) with larger activations in the right hemisphere, and right amygdala. In response to the perceived difference between stimuli, we found repetition effects in more widespread regions in- 
Table 1. Areas revealing significant modulations with physical and perceptual difference

\begin{tabular}{|c|c|c|c|c|c|c|}
\hline \multirow[b]{2}{*}{ Modulator } & \multirow[b]{2}{*}{ Anatomical definition } & \multicolumn{3}{|c|}{ MNI coordinates (peak voxel) } & \multirow[b]{2}{*}{$t / F$ value } & \multirow[b]{2}{*}{ Cluster size } \\
\hline & & $x$ & $y$ & $z$ & & \\
\hline \multirow[t]{9}{*}{ Physical difference } & Right superior temporal gyrus & 63 & -25 & 1 & 8.35 & 491 \\
\hline & Right superior temporal gyrus & 57 & -4 & -5 & 8.14 & \\
\hline & Right superior temporal gyrus & 57 & -13 & -2 & 7.73 & \\
\hline & Left superior temporal gyrus & -60 & -22 & 4 & 7.52 & 161 \\
\hline & Left superior temporal gyrus & -66 & -25 & 16 & 5.63 & \\
\hline & Left mid-temporal gyrus & -60 & -40 & 10 & 4.83 & \\
\hline & Right mid-cingulum & 12 & -46 & 34 & 6.27 & 36 \\
\hline & Right precuneus & 6 & -58 & 37 & 4.85 & \\
\hline & Right amygdala & 24 & -7 & -14 & 5.90 & 32 \\
\hline \multirow[t]{28}{*}{ Perceptual difference } & Left insula & -30 & 20 & 4 & 38.80 & 153 \\
\hline & Left precentral gyrus & -48 & 2 & 34 & 37.75 & 336 \\
\hline & Left rolandic operculum & -48 & 2 & 13 & 21.39 & \\
\hline & Left inferior frontal gyrus & -42 & 17 & 28 & 17.76 & \\
\hline & Left supplementary motor area & -3 & 14 & 49 & 32.90 & 205 \\
\hline & Right mid-cingulum & 9 & 26 & 31 & 13.68 & \\
\hline & Left precuneus & -3 & -67 & 31 & 32.44 & 516 \\
\hline & Left cuneus & -12 & -61 & 25 & 29.55 & \\
\hline & Left precuneus & -3 & -46 & 40 & 21.74 & \\
\hline & Right insula & 33 & 26 & 4 & 28.78 & 169 \\
\hline & Right insula & 39 & 20 & 1 & 27.09 & \\
\hline & Left superior temporal gyrus & -54 & -1 & -8 & 25.34 & 131 \\
\hline & Left mid-temporal & -51 & 5 & -23 & 18.61 & \\
\hline & Left temporal pole & -45 & 8 & -11 & 18.17 & \\
\hline & Right precentral gyrus & 45 & 2 & 34 & 25.28 & 240 \\
\hline & Right mid-frontal gyrus & 36 & 2 & 37 & 25.10 & \\
\hline & Right precentral gyrus & 48 & 2 & 43 & 19.77 & \\
\hline & Left superior frontal gyrus & -24 & 29 & 46 & 25.20 & 66 \\
\hline & Left mid-temporal gyrus & -48 & -76 & 22 & 23.73 & 241 \\
\hline & Left mid-occipital & -39 & -73 & 28 & 19.43 & \\
\hline & Left angular & -51 & -64 & 28 & 17.23 & \\
\hline & Right superior temporal gyrus & 54 & -19 & -2 & 21.52 & 75 \\
\hline & Right superior temporal gyrus & 45 & -25 & 1 & 14.79 & \\
\hline & Right superior temporal gyrus & 51 & -7 & -8 & 13.77 & \\
\hline & Right lingual & 9 & -82 & 4 & 20.21 & 83 \\
\hline & Right lingual & 15 & -70 & 7 & 16.12 & \\
\hline & Left medial superior frontal gyrus & -6 & 53 & 16 & 19.40 & 98 \\
\hline & Right medial superior frontal gyrus & 6 & 56 & 16 & 13.52 & \\
\hline
\end{tabular}

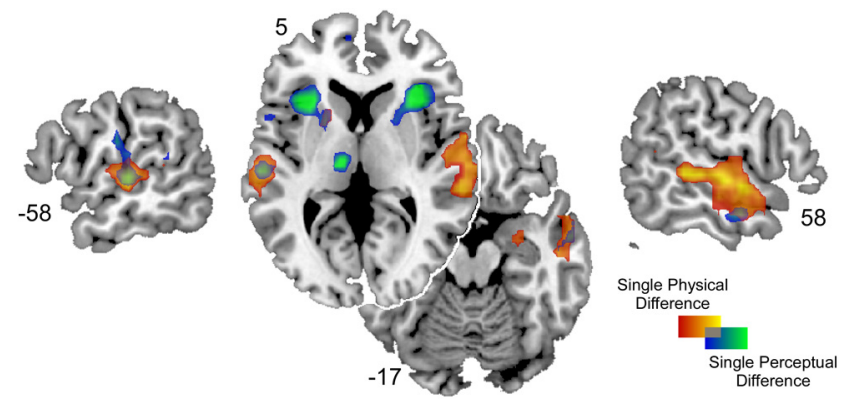

Figure 5. Overlap of the activation maps of significant correlations for physical (red-yellow) and perceptual (blue-green) difference regressors from the "single" models illustrated on a T1-weighted average structural template.

cluding bilateral anterior insulae, precentral gyri, and medial superior frontal areas, as well as amygdalae, hippocampi, and thalami at a more lenient threshold. Our study provides support for the key concept described in Schirmer and Kotz (2006) of multistep processes involved in the coding of auditory emotion but also highlights the unpredicted complexity of the network necessary for the evaluative judgments of affective vocalizations, even in the absence of speech.

Schirmer and Kotz (2006) propose a hierarchical model for the processing of emotional prosody (for a multimodal version, see also Wildgruber et al. (2009); Brück et al. (2011)). This model consists of three steps starting with a low-level acoustic analysis in bilateral auditory cortices followed by mid-level analyses in bilateral STS/STG, which synthesize emotionally salient information into an acoustic object. Finally, STS/STG then feed into frontal areas for higher-order cognition and evaluation. Most of the neuroimaging studies cited in support of the model used subtraction designs, which make a demonstration of the existence of separate steps and differential functions of STS/STG and IFGs difficult. Importantly, also, these studies do not typically differentiate activity patterns that process general physical differences between stimuli from those that process more abstract emotional information independent of lower-level acoustic changes. To our knowledge, only one study (Wiethoff et al., 2008) has used multiple regression to demonstrate that the passive-listening response to emotionally spoken words in the right mid-STG could be explained only by a combination of various acoustic parameters, but the study did not elucidate the areas responsible for the higher-level representation of emotion.

The continuous carry-over design allowed us to isolate the neural processes engaged in lower-level stages dealing with physical differences between sounds from higher-level stages involved in vocal affect cognition. Thus, we first computed correlations between the BOLD signal and the physical difference between morphs. Right amygdala as well as bilateral STS/STG, which 
Table 2. Areas revealing significant modulations with physical and perceptual difference from the single models

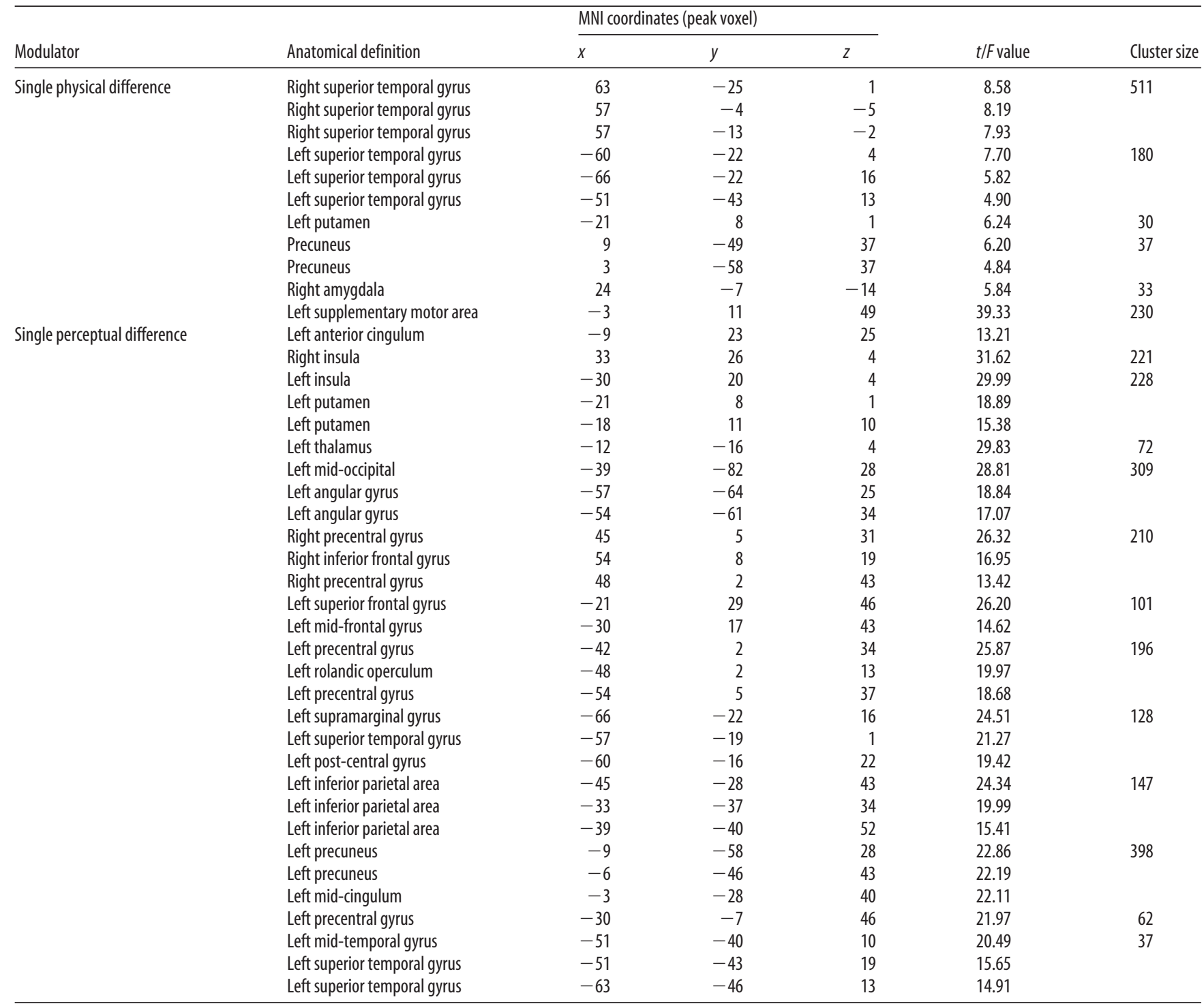

overlapped with voice-sensitive regions, correlated positively with an increased difference in physical properties between consecutive stimuli (for similar results with different task and design, see also Frühholz et al. (2012)). The bilateral correlation in voicesensitive cortex was lateralized to the right, confirming previous neuropsychological studies (Van Lancker and Sidtis, 1992; Hornak et al., 1996; Shamay-Tsoory et al., 2004; Ross and Monnot, 2008) and neuroimaging studies (Imaizumi et al., 1997; Morris et al., 1999; Buchanan et al., 2000; Rämä et al., 2001; Wildgruber et al., 2002; Mitchell et al., 2003; Grandjean et al., 2005; Wildgruber et al., 2005; Ethofer et al., 2006, 2009a,b; Leitman et al., 2010; Ethofer et al., 2012; for a recent meta-analysis, see Witteman et al., 2012) that provide support for the importance of these regions in the perception of emotional prosody. While STS/STG are classically highlighted in studies on affective prosody perception, they do not seem to be specific to the processing of auditory emotion, as activations in these areas have been reported for the perception of identity (Andics et al., 2010; Latinus et al., 2011, 2013), gender (Charest et al., 2013), and attractiveness (Bestelmeyer et al., 2012). Research on auditory object perception suggests that while subregions of the STG/STS may be specific to certain sound categories, these activations may not as such be indicative of representations of the semantic category but instead of a shared acoustic profile common to a particular group of auditory objects (e.g., vocalizations, musical instruments; Lewis et al. (2009); Leaver and Rauschecker, 2010). Thus, bilateral STS/ STG may be related to acoustic feature extraction of paralinguistic information more generally.

Second, to reveal the higher-level processes involved in vocal affect cognition, we computed correlations between the perceptual difference regressor (using an individual's behavioral data) and BOLD signal after the variance explained by the physical difference regressor had been explained. We found negative quadratic correlations in bilateral insulae (with IFG), mid-cingulum, superior frontal gyrus, and precentral gyrus, and a positive correlation in bilateral precuneus. The involvement of anterior insulae has frequently been reported in the perception of a variety of emotional, multimodal stimuli and experimental tasks (Duerden et al., 2013), emphasizing its importance in the processing and possible integration of emotional information from various senses. A recent review (Craig, 2009) reveals that the response of the anterior insulae is not specific to emotion because an astonishing number of studies covering a range of topics (e.g., emotional awareness, error awareness, and attention to pain) 
highlight activation of the anterior insulae with no consistent coactivation of other areas. The anterior insulae are therefore now thought of as a correlate of awareness or consciousness (Craig, 2009).

The precuneus and its functional significance have only recently received attention. Its anatomical location makes it hard to study, and it is therefore a comparatively less accurately mapped area of cortex (Cavanna and Trimble, 2006). The precuneus has widespread reciprocal corticocortical and subcortical connections. The principal connections outside parietal cortex are with the frontal lobes (including supplementary motor areas) and insulae, and it is noteworthy that the precuneus is not directly connected to any primary sensory area (Margulies et al., 2009). This pattern of connections implies that the precuneus is a major association area that may underpin a variety of different behaviors (Cavanna and Trimble, 2006). An inverse relationship between activity in anterior insulae and activity in precuneus has been associated with task-related attention (Dosenbach et al., 2007). We see a similar inverse pattern of activation in precuneus compared with anterior insulae and mid-cingulum, suggesting that these areas could be related to the attentional demands of the task rather than emotion perception per se. Importantly, albeit at a more lenient threshold, we observed activation in bilateral subcortical structures (amygdalae/hippocampi and thalami), all of which have been traditionally implicated in emotion perception.

The quadratic response to the perceptual difference was unexpected. In bilateral insulae, identical and maximally different morphs of precentral gyri and mid-cingulum produced lower responses than when it was less clear whether the morph belonged to the same category as the preceding one. Such complex response forms to continuous changes in stimulus features may be a property of higher-order association cortex and are an important topic for future investigation. While unexpected, these responses have been reported previously in visual psychophysics studies (Aguirre, 2007; Drucker et al., 2009) as well as a study on voice gender (Charest et al., 2013). Intuitively, these quadratic effects could be related to the experimental task, although these have been very different across studies (orthogonal vs active tasks).

We used nonverbal stimuli and a relatively simple task. Nevertheless our data suggest a dissociation between the processing of acoustic and cognitive aspects. It is plausible that the areas responsive to perceptual differences in affect are supramodal. In fact, a recent behavioral adaptation study suggests that there might be neurons which code expressions independently of modality (Skuk and Schweinberger, 2013). There are additional aspects which deserve future attention. First, the perceptual difference regressor was based on a simple categorization task and could be fine-tuned by incorporating more dimensions of emotion, e.g., intensity. We opted, in the first instance, to use data that can be obtained from the participant during scanning. Second, due to the nonverbal nature of our stimuli an interesting extension of this study could investigate the complex interactions between speech content and vocal affect. Furthermore, while our analytical approach was appropriate for teasing apart the processes dealing with acoustic and evaluative aspects of vocal expressions, it is limited in that it assumes our regressors of interest are orthogonal (when in fact they are to some extent correlated). As such, some of the variance attributed to the physical difference regressor is neither purely physical nor purely perceptual but shared. Therefore a strict dichotomy between cerebral effects driven by physical or perceptual features of the sounds is oversimplified.
Our results support the core idea described in Schirmer and Kotz (2006) that vocal affect recognition is a multistep process. Our findings extend that idea by identifying largely distinct regions of the emotion network for the coding of acoustic features of vocalization and for the processing of the cognitive representation of emotion. While the model originally proposes right IFG and orbitofrontal cortex as the hubs for evaluative judgments and left IFG for the processing of semantic content of emotional prosody, our findings highlight the involvement of a more complex, largely bilateral set of regions necessary to perform evaluative judgments of emotional vocalizations even in the absence of speech.

\section{References}

Aguirre GK (2007) Continuous carry-over designs for fMRI. Neuroimage 35:1480-1494. CrossRef Medline

Andics A, McQueen JM, Petersson KM, Gál V, Rudas G, Vidnyánszky Z (2010) Neural mechanisms for voice recognition. Neuroimage 52:1528 1540. CrossRef Medline

Belin P, Zatorre RJ (2000) "What," "where" and "how" in auditory cortex. Nat Neurosci 3:965-966. CrossRef Medline

Belin P, Zatorre RJ, Lafaille P, Ahad P, Pike B (2000) Voice-selective areas in human auditory cortex. Nature 403:309-312. CrossRef Medline

Belin P, Fillion-Bilodeau S, Gosselin F (2008) The Montreal Affective Voices: a validated set of nonverbal affect bursts for research on auditory affective processing. Behav Res Methods 40:531-539. CrossRef Medline

Bestelmeyer PE, Rouger J, DeBruine LM, Belin P (2010) Auditory adaptation in vocal affect perception. Cognition 117:217-223. CrossRef Medline

Bestelmeyer PE, Belin P, Grosbras MH (2011) Right temporal TMS impairs voice detection. Curr Biol 21:R838-R839. CrossRef Medline

Bestelmeyer PE, Latinus M, Bruckert L, Rouger J, Crabbe F, Belin P (2012) Implicitly perceived vocal attractiveness modulates prefrontal cortex activity. Cereb Cortex 22:1263-1270. CrossRef Medline

Brainard DH (1997) The psychophysics toolbox. Spat Vis 10:433-436. CrossRef Medline

Brück C, Kreifelts B, Wildgruber D (2011) Emotional voices in context: a neurobiological model of multimodal affective information processing. Phys Life Rev 8:383-403. CrossRef Medline

Buchanan TW, Lutz K, Mirzazade S, Specht K, Shah NJ, Zilles K, Jancke L (2000) Recognition of emotional prosody and verbal components of spoken language: an fMRI study. Cogn Brain Res 9:227-238. CrossRef

Büchel C, Holmes AP, Rees G, Friston KJ (1998) Characterizing stimulusresponse functions using nonlinear regressors in parametric fMRI experiments. Neuroimage 8:140-148. CrossRef Medline

Cavanna AE, Trimble MR (2006) The precuneus: a review of its functional anatomy and behavioural correlates. Brain 129:564-583. CrossRef Medline

Charest I, Pernet C, Latinus M, Crabbe F, Belin P (2013) Cerebral porcessing of voice gender studied using a continuous carryover FMRI design. Cereb Cortex 23:958-966. CrossRef Medline

Craig AD (2009) How do you feel-now? The anterior insula and human awareness. Nat Rev Neurosci 10:59-70. CrossRef Medline

Dosenbach NU, Fair DA, Miezin FM, Cohen AL, Wenger KK, Dosenbach RA, Fox MD, Snyder AZ, Vincent JL, Raichle ME, Schlaggar BL, Petersen SE (2007) Distinct brain networks for adaptive and stable task control in humans. Proc Natl Acad Sci U S A 104:11073-11078. CrossRef Medline

Drucker DM, Kerr WT, Aguirre GK (2009) Distinguishing conjoint and independent neural tuning for stimulus features with fMRI adaptation. J Neurophysiol 101:3310-3324. CrossRef Medline

Duerden EG, Arsalidou M, Lee M, Taylor MJ (2013) Lateralization of affective processing in the insula. Neuroimage 78:159-175. CrossRef Medline

Duvernoy HM (1999) The human brain: surface, blood supply, and threedimensional sectional anatomy. Vienna: Springer.

Ethofer T, Anders S, Erb M, Herbert C, Wiethoff S, Kissler J, Grodd W, Wildgruber D (2006) Cerebral pathways in processing of affective prosody: a dynamic causal modeling study. Neuroimage 30:580-587. CrossRef Medline

Ethofer T, Van De Ville D, Scherer K, Vuilleumier P (2009a) Decoding of emotional information in voice-sensitive cortices. Curr Biol 19:1028 1033. CrossRef Medline

Ethofer T, Kreifelts B, Wiethoff S, Wolf J, Grodd W, Vuilleumier P, Wildgru- 
ber D (2009b) Differential influences of emotion, task, and novelty on brain regions underlying the processing of speech melody. J Cogn Neurosci 21:1255-1268. CrossRef Medline

Ethofer T, Bretscher J, Gschwind M, Kreifelts B, Wildgruber D, Vuilleumier P (2012) Emotional voice areas: anatomic location, functional properties, and structural connections revealed by combined fMRI/DTI. Cereb Cortex 22:191-200. CrossRef Medline

Frühholz S, Ceravolo L, Grandjean D (2012) Specific brain networks during explicit and implicit decoding of emotional prosody. Cereb Cortex 22: 1107-1117. CrossRef Medline

Grandjean D, Sander D, Pourtois G, Schwartz S, Seghier ML, Scherer KR, Vuilleumier P (2005) The voices of wrath: brain responses to angry prosody in meaningless speech. Nat Neurosci 8:145-146. CrossRef Medline

Grill-Spector K, Kushnir T, Edelman S, Avidan G, Itzchak Y, Malach R (1999) Differential processing of objects under various viewing conditions in the human lateral occipital complex. Neuron 24:187-203. CrossRef Medline

Hornak J, Rolls ET, Wade D (1996) Face and voice expression identification inpatients with emotional and behavioural changes following ventral frontal lobe damage. Neuropsychologia 34:247-261. CrossRef Medline

Imaizumi S, Mori K, Kiritani S, Kawashima R, Sugiura M, Fukuda H, Itoh K, Kato T, Nakamura A, Hatano K, Kojima S, Nakamura K (1997) Vocal identification of speaker and emotion activates different brain regions. Neuroreport 8:2809-2812. CrossRef Medline

Kawahara H, Matsui H (2003) Auditory morphing based on an elastic perceptual distance metric in an interference-free time-frequency representation. Paper presented at IEEE International Conference on Acoustics, Speech, and Signal Processing, 2003, Hong Kong, People's Republic of China, April.

Latinus M, Crabbe F, Belin P (2011) Learning-induced changes in the cerebral processing of voice identity. Cereb Cortex 21:2820-2828. CrossRef Medline

Latinus M, McAleer P, Bestelmeyer PE, Belin P (2013) Norm-based coding of voice identity in human auditory cortex. Curr Biol 23:1075-1080. CrossRef Medline

Leaver AM, Rauschecker JP (2010) Cortical representation of natural complex sounds: effects of acoustic features and auditory object category. J Neurosci 30:7604-7612. CrossRef Medline

Leitman DI, Wolf DH, Ragland JD, Laukka P, Loughead J, Valdez JN, Javitt DC, Turetsky BI, Gur GC (2010) "It's not what you say, but how you say it": a reciprocal temporo-frontal network for affective prosody. Front Hum Neurosci 4:19. CrossRef Medline

Lewis JW, Talkington WJ, Walker NA, Spirou GA, Jajosky A, Frum C, Brefczynski-Lewis JA (2009) Human cortical organization for processing vocalizations indicates representation of harmonic structure as a signal attribute. J Neurosci 29:2283-2296. CrossRef Medline

Margulies DS, Vincent JL, Kelly C, Lohmann G, Uddin LQ, Biswal BB, Villringer A, Castellanos FX, Milham MP, Petrides M (2009) Precuneus shares intrinsic functional architecture in humans and monkeys. Proc Natl Acad Sci U S A 106:20069-20074. CrossRef Medline

Mitchell RL, Elliott R, Barry M, Cruttenden A, Woodruff PW (2003) The neural response to emotional prosody, as revealed by functional magnetic resonance imaging. Neuropsychologia 41:1410-1421. CrossRef Medline

Morris JS, Scott SK, Dolan RJ (1999) Saying it with feeling: neural responses to emotional vocalizations. Neuropsychologia 37:1155-1163. CrossRef Medline

Nonyane BA, Theobald CM (2007) Design sequences for sensory studies: achieving balance for carry-over and position effects. Br J Math Stat Psychol 60:339-349. CrossRef Medline

Patel S, Scherer KR, Björkner E, Sundberg J (2011) Mapping emotions into acoustic space: the role of voice production. Biol Psychol 87:93-98. CrossRef Medline

Pelli DG (1997) The VideoToolbox software for visual psychophysics: transforming numbers into movies. Spat Vis 10:437-442. CrossRef Medline

Rämä P, Martinkauppi S, Linnankoski I, Koivisto J, Aronen HJ, Carlson S (2001) Working memory of identification of emotional vocal expressions: an fMRI study. Neuroimage 13:1090-1101. CrossRef Medline

Rorden C, Karnath HO, Bonilha L (2007) Improving lesion-symptom mapping. J Cogn Neurosci 19:1081-1088. CrossRef Medline

Ross ED, Monnot M (2008) Neurology of affective prosody and its functional-anatomic organization in right hemisphere. Brain Lang 104: 51-74. CrossRef Medline

Schirmer A, Kotz SA (2006) Beyond the right hemisphere: brain mechanisms mediating vocal emotional processing. Trends Cogn Sci 10:24-30. CrossRef Medline

Schweinberger SR, Kawahara H, Simpson AP, Skuk VG, Zäske R (2014) Speaker perception. WIREs Cogn Sci 5:15-25. CrossRef

Shamay-Tsoory SG, Tomer R, Goldsher D, Berger BD, Aharon-Peretz J (2004) Impairment in cognitive and affective empathy in patients with brain lesions: anatomical and cognitive correlates. J Clin Exp Neuropsychol 26:1113-1127. CrossRef Medline

Skuk VG, Schweinberger SR (2013) Adaptation aftereffects in vocal emotion perception elicited by expressive faces and voices. PLoS One 8:e81691. CrossRef Medline

Van Lancker D, Sidtis JJ (1992) The identification fo affective-prosodic stimuli by left-hemisphere-damaged and right-hemisphere-damaged subjects—all errors are not created equal. J Speech Hear Res 35:963-970. Medline

Wallbott HG, Scherer KR (1986) Cues and channels in emotion recognition. J Pers Soc Psychol 51:690-699. CrossRef

Wiethoff S, Wildgruber D, Kreifelts B, Becker H, Herbert C, Grodd W, Ethofer T (2008) Cerebral processing of emotional prosody-influence of acoustic parameters and arousal. Neuroimage 39:885-893. CrossRef Medline

Wildgruber D, Pihan H, Ackermann H, Erb M, Grodd W (2002) Dynamic brain activation during processing of emotional intonation: influence of acoustic parameters, emotional valence, and sex. Neuroimage 15:856869. CrossRef Medline

Wildgruber D, Riecker A, Hertrich I, Erb M, Grodd W, Ethofer T, Ackermann $\mathrm{H}$ (2005) Identification of emotional intonation evaluated by fMRI. Neuroimage 24:1233-1241. CrossRef Medline

Wildgruber D, Ethofer T, Grandjean D, Kreifelts B (2009) A cerebral network model of speech prosody comprehension. Int J Speech Lang Pathol 11:277-281. CrossRef

Witteman J, Van Heuven VJ, Schiller NO (2012) Hearing feelings: a quantitative meta-analysis on the neuroimaging literature of emotional prosody perception. Neuropsychologia 50:2752-2763. CrossRef Medline 